\title{
On cyclic Schur-positive sets of permutations
}

\author{
Jonathan Bloom* \\ Department of Mathematics \\ Lafayette College \\ Easton, PA 18042, U.S.A. \\ bloomjs@lafayette.edu
}

\author{
Sergi Elizalde ${ }^{\dagger}$ \\ Department of Mathematics \\ Dartmouth College \\ Hanover, NH 03755, U.S.A.
}

sergi.elizalde@dartmouth.edu

\author{
Yuval Roichman \\ Department of Mathematics \\ Bar-Ilan University \\ Ramat-Gan 52900, Israel \\ yuvalr@math.biu.ac.il
}

Submitted: Aug 28, 2019; Accepted: Mar 12, 2020; Published: Apr 17, 2020

(C) The authors. Released under the CC BY-ND license (International 4.0).

\begin{abstract}
We introduce a notion of cyclic Schur-positivity for sets of permutations, which naturally extends the classical notion of Schur-positivity, and it involves the existence of a bijection from permutations to standard Young tableaux that preserves the cyclic descent set. Cyclic Schur-positive sets of permutations are always Schurpositive, but the converse does not hold, as exemplified by inverse descent classes, Knuth classes and conjugacy classes.

In this paper we show that certain classes of permutations invariant under either horizontal or vertical rotation are cyclic Schur-positive. The proof unveils a new equidistribution phenomenon of descent sets on permutations, provides affirmative solutions to conjectures by the last two authors and by Adin-Gessel-ReinerRoichman, and yields new examples of Schur-positive sets.
\end{abstract}

Mathematics Subject Classifications: 05E05

\footnotetext{
* Partially supported by a Bar-Ilan University visiting grant.

${ }^{\dagger}$ Partially supported by Simons Foundation grant \#280575. Corresponding author.

$\ddagger$ Partially supported by an MIT-Israel MISTI grant and by the Israel Science Foundation, grant no. 1970/18.
} 


\section{Introduction}

Let $[n]:=\{1,2, \ldots, n\}$ and let $\mathfrak{S}_{n}$ denote the symmetric group on $[n]$. Recall that the descent set of a permutation $\pi \in \mathfrak{S}_{n}$ is

$$
\operatorname{Des}(\pi):=\{i \in[n-1]: \pi(i)>\pi(i+1)\} .
$$

Given any subset $A \subseteq \mathfrak{S}_{n}$, we define the quasi-symmetric function

$$
\mathcal{Q}(A):=\sum_{\pi \in A} \mathcal{F}_{n, \operatorname{Des}(\pi)},
$$

where $\mathcal{F}_{n, D}$ is Gessel's fundamental quasi-symmetric function [11], defined for $D \subseteq[n-1]$ by

$$
\mathcal{F}_{n, D}:=\sum_{\substack{i_{1} \leqslant i_{2} \leqslant \cdots \leqslant i_{n} \\ i_{j}<i_{j+1} \text { if } j \in D}} x_{i_{1}} x_{i_{2}} \cdots x_{i_{n}} .
$$

A symmetric function is called Schur-positive if all the coefficients in its expansion in the basis of Schur functions are nonnegative. A subset $A \subseteq \mathfrak{S}_{n}$ is called Schur-positive if $\mathcal{Q}(A)$ is symmetric and Schur-positive.

The following long-standing problem was first addressed in [12].

Problem 1. Find Schur-positive subsets of $\mathfrak{S}_{n}$.

It is possible to characterize Schur-positive permutation sets using standard Young tableaux (SYT). We write $\lambda / \mu \vdash n$ to mean that $\lambda / \mu$ is a skew shape with $n$ boxes, where $\lambda$ and $\mu$ are partitions such that the Young diagram of $\mu$ is contained in that of $\lambda$. Let $\operatorname{SYT}(\lambda / \mu)$ denote the set of standard Young tableaux of shape $\lambda / \mu$. We draw tableaux in English notation, as in Figure 1. The descent set of $T \in \operatorname{SYT}(\lambda / \mu)$ is

$$
\operatorname{Des}(T):=\{i \in[n-1]: i+1 \text { is in a lower row than } i \text { in } T\} .
$$

For example, the descent set of the SYT in Figure 1 is $\{1,4,5,8\}$. For $J \subseteq[n-1]$, let $\mathbf{t}^{J}:=\prod_{i \in J} t_{i}$

$$
\begin{array}{|l|l|l|l|l|}
\hline 1 & 3 & 4 & 8 \\
\hline & 2 & 5 & 7 & \multicolumn{1}{|c}{} \\
\cline { 1 - 3 } 6 & 9 & \multicolumn{3}{|c}{} \\
\cline { 1 - 2 } & & \multicolumn{3}{|c}{} \\
\hline
\end{array}
$$

Figure 1: A SYT of shape $(5,4,2) /(1,1)$.

Theorem 2 ([4, Prop. 9.1]). A subset $A \subseteq \mathfrak{S}_{n}$ is Schur-positive if and only if there exist nonnegative integers $\left(b_{\lambda}\right)_{\lambda \vdash n}$ such that

$$
\sum_{\pi \in A} \mathbf{t}^{\operatorname{Des}(\pi)}=\sum_{\lambda \vdash n} b_{\lambda} \sum_{T \in \operatorname{SYT}(\lambda)} \mathbf{t}^{\operatorname{Des}(T)} .
$$


This characterization of Schur-positive sets of permutations is useful because it does not require computing quasisymmetric functions, but rather finding a Des-preserving bijection from permutations to SYT of shapes given by a certain multiset.

In this paper we introduce and study a cyclic analogue of Schur-positive permutation sets, whose definition is motivated by Theorem 2. Before we state Definition 4, we need some background on cyclic descent sets.

The cyclic descent set for permutations was introduced by Cellini [6] and later studied by Dilks, Petersen, Stembridge [7], and others. For $\pi \in \mathfrak{S}_{n}$ let

$$
\operatorname{cDes}(\pi):=\{i \in[n]: \pi(i)>\pi(i+1)\},
$$

with the convention $\pi(n+1):=\pi(1)$.

Example 3. For $\pi=21453 \in \mathfrak{S}_{5}$, we have $\operatorname{Des}(\pi)=\{1,4\}$ and $\operatorname{cDes}(\pi)=\{1,4,5\}$.

The cyclic descent set for rectangular SYT was introduced by Rhoades [15], and extended to some other shapes in $[1,10]$. This notion was generalized to all skew shapes that are not connected ribbons in [3]. An explicit combinatorial description of cyclic descent sets on $\operatorname{SYT}(\lambda / \mu)$ for every skew shape which is not a connected ribbon was recently given by Huang [13]. The following is the main definition in this paper.

Definition 4. A subset $A \subseteq \mathfrak{S}_{n}$ is cyclic Schur-positive $(c S p)$ if there exists a collection of nonnegative integers $\left(b_{\lambda / \mu}\right)_{\lambda / \mu \vdash n}$ such that

$$
\sum_{\pi \in A} \mathbf{t}^{\mathrm{cDes}(\pi)}=\sum_{\lambda / \mu \vdash n} b_{\lambda / \mu} \sum_{T \in \operatorname{SYT}(\lambda / \mu)} \mathbf{t}^{\mathrm{cDes}(T)},
$$

where $\operatorname{cDes}(\pi)$ is defined by Eq. $(4), \operatorname{cDes}(T)$ is the cyclic descent set defined in $[3,13]$, and the sum in the RHS is over skew shapes $\lambda / \mu$ that are not connected ribbons (and thus cyclic descent sets exist for SYT of these shapes).

In Theorem 15 we give an alternative characterization of cyclic Schur-positive permutation sets using an invariance property of cyclic descent sets.

It will follow from this characterization that cyclic Schur-positive sets of permutations are always Schur-positive. However, the converse does not hold; in fact, most known examples of Schur-positive sets are not cyclic Schur-positive. One of our goals is to address the following problem.

Problem 5. Find cyclic Schur-positive (cSp) subsets of $\mathfrak{S}_{n}$.

In this paper we present two families of cSp sets of permutations: horizontal rotations of Schur-positive sets, and vertical rotations of inverse descent classes, which include arc permutations. Let us now define these concepts.

To define horizontal and vertical rotations, let $c_{n}$ denote the $n$-cycle $(1,2, \ldots, n)=$ $23 \ldots n 1 \in \mathfrak{S}_{n}$, and let $C_{n}:=\left\langle c_{n}\right\rangle$ be the cyclic subgroup generated by $c_{n}$. 
Any set $A \subseteq \mathfrak{S}_{n-1}$ can be interpreted as a subset of $\mathfrak{S}_{n}$ by identifying $\mathfrak{S}_{n-1}$ with the set of permutations in $\mathfrak{S}_{n}$ that fix $n$. With this interpretation, we define the horizontal (respectively, vertical) rotation closure of $A \subseteq \mathfrak{S}_{n-1}$ as the set $A C_{n} \subseteq \mathfrak{S}_{n}$ (respectively, $C_{n} A \subseteq \mathfrak{S}_{n}$ ). Our first main result (Theorem 23) states that if $A \subseteq \mathfrak{S}_{n-1}$ is Schurpositive, then its horizontal rotation closure $A C_{n} \subseteq \mathfrak{S}_{n}$ is cSp. As a consequence, we show in Corollary 24 that the set of permutations whose inverses have a given number of cyclic descents is cSp.

For every $J \subseteq[n-2]$, define the descent class

$$
D_{n-1, J}:=\left\{\pi \in \mathfrak{S}_{n-1}: \operatorname{Des}(\pi)=J\right\} .
$$

The inverse of a set of permutations $A \subseteq \mathfrak{S}_{n}$ is defined as $A^{-1}:=\left\{\pi^{-1}: \pi \in A\right\}$. Our second main result (Theorem 25) implies that for any inverse descent class, the distribution of the statistic cDes is the same on its vertical rotations $C_{n} D_{n-1, J}^{-1}$ as on its horizontal rotations $D_{n-1, J}^{-1} C_{n}$. The proof of this result, which settles a stronger version of [9, Conjecture 10.2], involves cDes-preserving operations on grid classes, as defined in [5]. Even though our proof is not bijective in general, we give explicit cDes-preserving bijections between $D_{n-1, J}^{-1} C_{n}$ and $C_{n} D_{n-1, J}^{-1}$ when $|J|=1$ in Section 5 , and when $J=[i]$ in Section 6.

Combining the two main theorems mentioned above, it follows that the set $C_{n} D_{n-1, J}^{-1}$ of vertical rotations of an inverse descent class is cSp (Theorem 33). In particular, it is Schur-positive, as had been conjectured in [9].

Of special interest is the set $\mathcal{A}_{n}$ of arc permutations, which are those permutations in $\mathfrak{S}_{n}$ where every prefix forms an interval in $\mathbb{Z}_{n}$. As we will see in Section 6 , this set is a union of vertical rotations of inverse descent classes, so we deduce (Corollary 42) that it is cSp as well, and we provide a bijective proof of this fact (Theorem 50).

\section{Background}

\subsection{Schur-positive permutation sets}

Recall that $A \subseteq \mathfrak{S}_{n}$ is Schur-positive if the quasi-symmetric function $\mathcal{Q}(A)$ from Equation (2) is symmetric and Schur-positive. A direct combinatorial characterization of Schurpositive sets was described in Theorem 2 .

Some classical examples of Schur-positive sets of permutations are given in Table 1. Other examples have appeared more recently in $[8,9,10]$.

\subsection{Cyclic descents for SYT}

Originally introduced by Rhoades [15] in the setting of rectangular shapes and later extended to some other shapes in $[1,14]$, the notion of cyclic descent set of SYT was defined for arbitrary skew shapes in [3]. The following definition, introduced in [1,3], was motivated by the basic common properties of cyclic descent sets of permutations, defined in Equation (4), and of SYT in the cases for which the definition was known at that time. For 


\begin{tabular}{l|l|l} 
Schur-positive subset of $\mathfrak{S}_{n}$ & Reference & Related examples \\
\hline Knuth class & {$[11]$} & $\begin{array}{l}\text { subsets invariant under Knuth rela- } \\
\text { tions (e.g. inverse descent classes, } \\
321 \text {-avoiding permutations) }\end{array}$ \\
\hline $\begin{array}{l}\text { conjugacy class } \\
\begin{array}{l}\text { permutations with a fixed in- } \\
\text { version number }\end{array}\end{array}$ & {$[1$, Prop. 9.5 $]$} & $\begin{array}{l}\text { subsets invariant under conjugation } \\
\text { (e.g. involutions) }\end{array}$ \\
\hline
\end{tabular}

Table 1: Classical examples of Schur-positive sets of permutations

a set $D \subseteq[n]$ and an integer $i$, we use the notation $i+D:=\{i+d \bmod n: d \in D\} \subseteq[n]$. Throughout the paper, addition of elements in $[n]$ will be interpreted modulo $n$.

Definition 6. Let $\mathcal{T}$ be a finite set. A descent map is any map Des : $\mathcal{T} \longrightarrow 2^{[n-1]}$. A cyclic extension of Des (also called a cyclic descent extension) is a pair (cDes, $\psi$ ), where cDes $: \mathcal{T} \longrightarrow 2^{[n]}$ is a map (called a cyclic descent map) and $\psi: \mathcal{T} \longrightarrow \mathcal{T}$ is a bijection, satisfying the following axioms: for all $T$ in $\mathcal{T}$,

$$
\begin{aligned}
\text { (extension) } & \mathrm{cDes}(T) \cap[n-1]=\operatorname{Des}(T), \\
\text { (equivariance) } & \mathrm{cDes}(\psi(T))=1+\operatorname{cDes}(T), \\
\text { (non-Escher) } & \emptyset \subsetneq \mathrm{cDes}(T) \subsetneq[n] .
\end{aligned}
$$

When $\mathcal{T}$ is a set of SYT, it will be assumed that the descent map Des is the one defined in Equation (3). Similarly, when $\mathcal{T}$ is a set of permutations, Des and cDes will refer to the maps defined in Equations (1) and (4), respectively.

Theorem 7 ([3, Theorem 1.1]). Let $\lambda / \mu$ be a skew shape. There exists a cyclic descent extension for $\operatorname{SYT}(\lambda / \mu)$ if and only if $\lambda / \mu$ is not a connected ribbon.

An explicit combinatorial description of a cyclic descent extension on $\operatorname{SYT}(\lambda / \mu)$ for every skew shape which is not a connected ribbon was recently given by Huang [13].

Example 8. Write $\mu^{1} \oplus \mu^{2} \oplus \cdots \oplus \mu^{t}$ to denote the skew shape consisting of $t$ connected components $\mu^{1}, \ldots, \mu^{t}$, ordered from southwest to northeast. A strip is a shape $\mu^{1} \oplus \mu^{2} \oplus$ $\cdots \oplus \mu^{t}$, each of whose connected components has either only one row or only one column. For a SYT $T$ of a strip shape with at least 2 components, let

$$
\operatorname{cDes}(T)= \begin{cases}\operatorname{Des}(T) \sqcup\{n\} & \text { if } 1 \text { is lower than } n, \\ & \text { or } 1 \text { and } n \text { are in the same vertical component } \\ \operatorname{Des}(T) & \text { otherwise. }\end{cases}
$$

Let $\psi(T):=1+T$, where $j+T$ is the SYT obtained by adding $j$ to each entry of $T$, modulo $n$, then rearranging the letters within each component in increasing order from left to right (if the component is a row) or from top to bottom (if it is a column). Note 
that $\psi^{j}(T)=j+T$ for all $j$. As shown in [1, Prop. 4.1], $\mathrm{cDes}(1+T)=1+\operatorname{cDes}(T)$, so $(\mathrm{cDes}, \psi)$ is a cyclic descent extension.

For example, letting

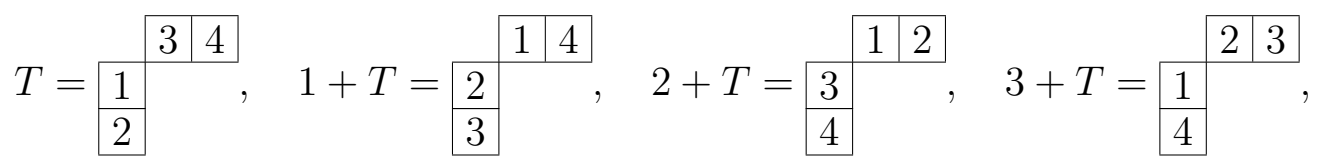

the corresponding cyclic descent sets are $\{1,4\},\{1,2\},\{2,3\}$ and $\{3,4\}$, respectively.

In the special case where SYT is a horizontal strip shape with at least 2 components, we have

$$
\operatorname{cDes}(T):=\{i \in[n]: i+1 \bmod n \text { is in a lower row than } i \text { in } T\}
$$

For example, letting

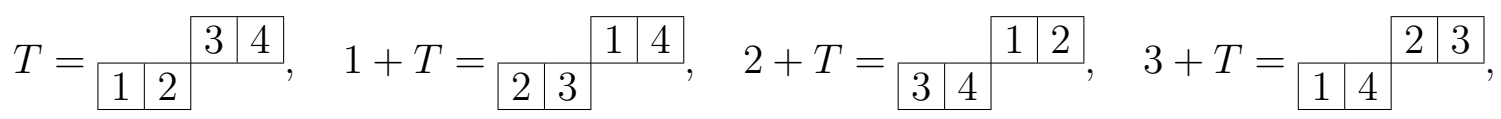

the corresponding cyclic descent sets are $\{4\},\{1\},\{2\}$ and $\{3\}$, respectively.

Example 9. By [1, Corollary 3.9], for every $0 \leqslant k<n-1$, there exists a unique cyclic descent map on $\operatorname{SYT}\left(\left(n-1-k, 1^{k}\right) \oplus(1)\right)$, specified by letting

$$
\operatorname{cDes}(T)= \begin{cases}\operatorname{Des}(T) \sqcup\{n\} & \text { if }|\operatorname{Des}(T)|=k, \\ \operatorname{Des}(T) & \text { otherwise, }\end{cases}
$$

for every $T \in \operatorname{SYT}\left(\left(n-1-k, 1^{k}\right) \oplus(1)\right)$. Denote by $\delta(T)$ the entry in the northeast disconnected cell of such tableau.

For $0<j<n$, let $j+T$ be the SYT obtained by letting the set of entries in the first column (excluding the corner cell) be $j+1+(\operatorname{cDes}(T) \backslash \delta(T))$ if $n-j \notin \operatorname{Des}(T)$, and $j+1+(\operatorname{cDes}(T) \backslash\{n-j\})$ if $n-j \in \operatorname{Des}(T)$, and letting $\delta(j+T):=j+\delta(T)$. By $[1$, Lemma 4.7], $\operatorname{cDes}(j+T)=j+\mathrm{cDes}(T)$, so a cyclic descent extension is obtained by taking $\psi(T):=1+T$.

The following lemma is used in Section 3.

Lemma 10 ([3, Lemma 2.2]). Let $\mathcal{T}$ be a finite set which carries a descent map Des : $\mathcal{T} \longrightarrow 2^{[n-1]}$. Assume that there exists a cyclic descent extension (cDes, $\psi$ ). Then, for every subset $\emptyset \neq J=\left\{j_{1}<\cdots<j_{t}\right\} \subseteq[n]$, the fiber size $\left|\operatorname{cDes}^{-1}(J)\right|$ is uniquely determined by the formula

$$
\left|\operatorname{cDes}^{-1}(J)\right|=\sum_{i=1}^{t}(-1)^{i-1}\left|\operatorname{Des}^{-1}\left(\left\{j_{i+1}-j_{i}, \ldots, j_{t}-j_{i}\right\}\right)\right|,
$$

where we interpret $\left\{j_{i+1}-j_{i}, \ldots, j_{t}-j_{i}\right\}$ as $\emptyset$ when $i=t$. 


\section{Cyclic Schur-positive permutation sets}

\subsection{Basic examples}

Definition 4 introduces the concept of cyclic Schur-positivity (cSp), which is the central notion of this paper. Let us make some remarks about this definition. First, note the analogy with Theorem 2, which characterizes Schur-positivity of sets of permutations. One difference, however, is the use of skew shapes in Definition 4. This modification is needed because SYT of hook shapes do not carry a cyclic descent extension.

Second, note that Lemma 10 implies that, for every skew shape $\lambda / \mu$ which is not a connected ribbon, the polynomial

$$
\sum_{T \in \operatorname{SYT}(\lambda / \mu)} \mathbf{t}^{\operatorname{cDes}(T)}
$$

is well defined, in the sense that it does not depend on the choice of cDes.

Example 11. Recall the cyclic subgroup $C_{n}$ generated by the $n$-cycle $c_{n}=(1,2, \ldots, n)$. By the definitions in Example 8, we have

$$
\sum_{\pi \in C_{n}} \mathbf{t}^{\mathrm{cDes}(\pi)}=\sum_{i=1}^{n} t_{i}=\sum_{T \in \operatorname{SYT}((1) \oplus(n-1))} \mathbf{t}^{\mathrm{cDes}(T)},
$$

thus $C_{n}$ is cSp.

Example 12. By [3, Theorem 1.2],

$$
\begin{aligned}
& \sum_{\pi \in \mathfrak{S}_{n}} \mathbf{t}^{\mathrm{cDes}(\pi)} \\
& \quad=\sum_{\substack{\text { non-hook } \\
\lambda \vdash n}}|\operatorname{SYT}(\lambda)| \sum_{T \in \operatorname{SYT}(\lambda)} \mathbf{t}^{\mathrm{cDes}(T)}+\sum_{k=1}^{n-1}\left(\begin{array}{c}
n-2 \\
k-1
\end{array}\right) \sum_{T \in \operatorname{SYT}\left(\left(n-k+1,1^{k}\right) /(1)\right)} \mathbf{t}^{\mathrm{cDes}(T)},
\end{aligned}
$$

thus $\mathfrak{S}_{n}$ is cSp.

\subsection{An alternative characterization}

The following equivariance property will be used to give an alternative characterization of cSp sets.

Definition 13. A subset $A \subseteq \mathfrak{S}_{n}$ is called cDes-invariant if there exists a bijection $\psi: A \rightarrow A$ such that, for all $\pi \in A$,

$$
\operatorname{cDes}(\psi \pi)=1+\operatorname{cDes}(\pi) .
$$

Remark 14. A subset $A \subseteq \mathfrak{S}_{n}$ is invariant under horizontal rotation if $A=A c_{n}$, where $c_{n}=(1,2, \ldots, n)$. Subsets invariant under horizontal rotation are cDes-invariant, since one can simply take $\psi \pi=\pi c_{n}^{-1}$ in Definition 13. 
The following characterization of cSp sets will be used in the rest of the paper.

Theorem 15. A subset $A \subseteq \mathfrak{S}_{n}$ is cyclic Schur-positive if and only if it is Schur-positive and cDes-invariant.

Before proving this theorem, let us consider some examples of its usage.

Example 16. The set $C_{n}=\left\langle c_{n}\right\rangle$ satisfies

$$
\sum_{\pi \in C_{n}} \mathbf{t}^{\operatorname{Des}(\pi)}=1+\sum_{i=1}^{n-1} t_{i}=\sum_{T \in \operatorname{SYT}((1) \oplus(n-1))} \mathbf{t}^{\operatorname{Des}(\pi)}=\sum_{T \in \operatorname{SYT}(n-1,1) \cup \operatorname{SYT}(n)} \mathbf{t}^{\operatorname{Des}(\pi)} .
$$

Thus, by Theorem 2, it is Schur-positive. On the other hand, by Remark 14, $C_{n}$ is cDes-invariant. Hence, by Theorem 15, $C_{n}$ is cSp, in agreement with Example 11.

Example 17. The Knuth class $K=\{2143,2413\} \subset \mathfrak{S}_{4}$, corresponding to the tableau \begin{tabular}{|l|l|}
\hline 1 & 3 \\
\hline 2 & 4 \\
\hline
\end{tabular} , is Schur-positive, since

$$
\sum_{\pi \in K} \mathbf{t}^{\operatorname{Des}(\pi)}=t_{1} t_{3}+t_{2}=\sum_{Q \in \operatorname{SYT}(2,2)} \mathbf{t}^{\operatorname{Des}(Q)}
$$

However, $K$ is not cDes-invariant, because $\operatorname{cDes}(2143)=\{1,3,4\}$ and $\operatorname{cDes}(2413)=$ $\{2,4\}$. Thus, it is not cSp.

Remark 18. By Theorem 15, every cSp set of permutations is Schur-positive. The converse does not hold, as Example 17 shows.

We now turn our attention to the proof of Theorem 15. In the next two lemmas, we assume that $A \subseteq \mathfrak{S}_{n}$ is Schur-positive and cDes-invariant, and we let $\left(b_{\lambda}\right)_{\lambda \vdash n}$ be nonnegative integers such that

$$
\sum_{\pi \in A} \mathbf{t}^{\operatorname{Des}(\pi)}=\sum_{\lambda \vdash n} b_{\lambda} \sum_{T \in \operatorname{SYT}(\lambda)} \mathbf{t}^{\operatorname{Des}(T)}
$$

which exist by Theorem 2 .

Lemma 19. For every $0 \leqslant k<n$,

$$
b_{\left(n-k, 1^{k}\right)}=|\{a \in A: \operatorname{Des}(a)=[k]\}|,
$$

where we define $[0]:=\emptyset$.

Proof. For every $0 \leqslant k<n$, there is a unique SYT $T$ with $\operatorname{Des}(T)=[k]$, namely the tableau of shape $\left(n-k, 1^{k}\right)$ having $1, \ldots, k+1$ in the first column and $1, k+2, \ldots, n$ in the first row. Comparing the coefficients of $\mathbf{t}^{[k]}$ on both sides of Equation (10) completes the proof. 
Lemma 20. For every $0 \leqslant k<n$, the alternating sum

$$
\sum_{i=k}^{n-1}(-1)^{k-i} b_{\left(n-i, 1^{i}\right)}
$$

is nonnegative; when $k=0$, it is zero.

Proof. To simplify notation, let us first write, for any $S \subseteq[n]$,

$$
A_{S}:=\{a \in A \mid \operatorname{cDes}(a)=S\} .
$$

By Lemma 19, for $0 \leqslant k<n$,

$$
b_{\left(n-i, 1^{i}\right)}=|\{a \in A: \operatorname{Des}(a)=[i]\}|=\left|A_{[i]}\right|+\left|A_{[i] \cup\{n\}}\right| .
$$

Since $A$ is cDes-invariant, we have $\left|A_{[i] \cup\{n\}}\right|=\left|A_{[i+1]}\right|$. Combining these facts we see that

$$
\sum_{i=k}^{n-1}(-1)^{k-i} b_{\left(n-i, 1^{i}\right)}=\sum_{i=k}^{n-1}(-1)^{k-i}\left(\left|A_{[i]}\right|+\left|A_{[i] \cup\{n\}}\right|\right)=\sum_{i=k}^{n-1}(-1)^{k-i}\left(\left|A_{[i]}\right|+\left|A_{[i+1]}\right|\right) .
$$

The telescoping sum on the right-hand side further reduces to

$$
\left|A_{[k]}\right|+(-1)^{k-n+1}\left|A_{[n]}\right|=\left|A_{[k]}\right|,
$$

using that $A_{[n]}=\emptyset$ by the non-Escher property. This expression is clearly nonnegative, and it equals zero when $k=0$, since $A_{[0]}=\emptyset$ again by the non-Escher property.

Lemma 21. Let $A \subseteq \mathfrak{S}_{n}$ be cDes-invariant. Assume that there are nonnegative constants $\left(b_{\lambda / \mu}\right)_{\lambda / \mu \vdash n}$ such that

$$
\sum_{\pi \in A} \mathbf{t}^{\operatorname{Des}(\pi)}=\sum_{\lambda / \mu \vdash n} b_{\lambda / \mu} \sum_{T \in \operatorname{SYT}(\lambda / \mu)} \mathbf{t}^{\operatorname{Des}(T)} .
$$

Further, assume that $b_{\lambda / \mu}=0$ whenever $\lambda / \mu$ is a connected ribbon. Then $A$ is $c S p$.

Proof. Let $B(\mathbf{t})$ be the quantity in Equation (11). As no ribbon shape appears with positive multiplicity on the right-hand side, we know from Theorem 7 that a cyclic descent extension (cDes, $\psi$ ) exists so that

$$
D(\mathbf{t}):=\sum_{\lambda / \mu \vdash n} b_{\lambda / \mu} \sum_{T \in \operatorname{SYT}(\lambda / \mu)} \mathbf{t}^{\mathrm{cDes}(T)}
$$

is a well-defined quantity. Set

$$
C(\mathbf{t}):=\sum_{\pi \in A} \mathbf{t}^{\mathrm{cDes}(\pi)},
$$

where cDes is now the cyclic descent for permutations given by Equation (4). 
By definition, this cyclic descent for permutations satisfies the extension and nonEscher properties from Definition 6. In addition, since $A$ is cDes-invariant, there exists a bijection $\rho: A \rightarrow A$ through which cDes satisfies the equivariance property. Thus, the pair (cDes, $\rho$ ) is a cyclic descent extension on $A$.

For every subset $\emptyset \neq J=\left\{j_{1}<\cdots<j_{t}\right\} \subseteq[n]$, let $b(J), c(J)$ and $d(J)$ be the coefficients of $\mathbf{t}^{J}$ in $B(\mathbf{t}), C(\mathbf{t})$ and $D(\mathbf{t})$, respectively. It follows from Lemma 10 that

$$
c(J)=\sum_{i=1}^{t}(-1)^{i-1} b\left(\left\{j_{i+1}-j_{i}, \ldots, j_{t}-j_{i}\right\}\right)=d(J) .
$$

Thus $C(\mathbf{t})=D(\mathbf{t})$, which implies that $A$ is cSp.

Proof of Theorem 15. For $0 \leqslant k \leqslant n$, we have

$$
\sum_{T \in \operatorname{SYT}\left(1^{k} \oplus(n-k)\right)} \mathbf{t}^{\operatorname{Des}(T)}=\sum_{T \in \operatorname{SYT}\left(n-k, 1^{k}\right)} \mathbf{t}^{\operatorname{Des}(T)}+\sum_{T \in \operatorname{SYT}\left(n-k+1,1^{k-1}\right)} \mathbf{t}^{\operatorname{Des}(T)} .
$$

Indeed, each tableau in $\operatorname{SYT}\left(1^{k} \oplus(n-k)\right)$ where the leftmost value in the first row is smaller (respectively, larger) than the top value in the first column corresponds to a tableau in $\operatorname{SYT}\left(n-k, 1^{k}\right)$ (respectively, $\operatorname{SYT}\left(n-k+1,1^{k-1}\right)$ ). Solving for the first term on the right-hand side and iterating the resulting equality, we get

$$
\sum_{T \in \operatorname{SYT}\left(n-k, 1^{k}\right)} \mathbf{t}^{\operatorname{Des}(T)}=\sum_{i=0}^{k}(-1)^{k-i} \sum_{T \in \operatorname{SYT}\left(1^{i} \oplus(n-i)\right)} \mathbf{t}^{\operatorname{Des}(T)} .
$$

Now assume that $A$ is Schur-positive and cDes-invariant, and let $b_{\lambda}$ be given by Equation (10). By Equality (13), the contribution from hook shapes to the right-hand side of Equation (10) is

$$
\begin{aligned}
\sum_{k=0}^{n-1} b_{\left(n-k, 1^{k}\right)} \sum_{T \in \operatorname{SYT}\left(n-k, 1^{k}\right)} \mathbf{t}^{\operatorname{Des}(T)} & =\sum_{k=0}^{n-1} b_{\left(n-k, 1^{k}\right)} \sum_{i=0}^{k}(-1)^{k-i} \sum_{T \in \operatorname{SYT}\left(1^{i} \oplus(n-i)\right)} \mathbf{t}^{\operatorname{Des}(T)} \\
& =\sum_{i=0}^{n-1} d_{i} \sum_{T \in \operatorname{SYT}\left(1^{i} \oplus(n-i)\right)} \mathbf{t}^{\operatorname{Des}(T)}
\end{aligned}
$$

interchanging the order of summation and letting $d_{i}:=\sum_{k=i}^{n-1}(-1)^{k-i} b_{\left(n-k, 1^{k}\right)}$. By Lemma 20, the coefficients $d_{0}, \ldots, d_{n-1}$ are nonnegative integers and $d_{0}=0$. One can now rewrite Equation (10) as

$$
\sum_{\pi \in A} \mathbf{t}^{\operatorname{Des}(\pi)}=\sum_{\substack{\lambda \vdash n \\ \lambda \text { not a hook }}} b_{\lambda} \sum_{T \in \operatorname{SYT}(\lambda)} \mathbf{t}^{\operatorname{Des}(T)}+\sum_{i=1}^{n-2} d_{i} \sum_{T \in \operatorname{SYT}\left(1^{i} \oplus(n-i)\right)} \mathbf{t}^{\operatorname{Des}(T)} .
$$


Observe that none of the shapes on the right-hand side are ribbon shapes. It now follows from Lemma 21 that $A$ is cSp.

For the converse, assume now that $A$ is cSp. Setting $t_{n}=1$ in Equation (5) gives

$$
\sum_{\pi \in A} \mathbf{t}^{\operatorname{Des}(\pi)}=\sum_{\lambda / \mu \vdash n} b_{\lambda / \mu} \sum_{T \in \operatorname{SYT}(\lambda / \mu)} \mathbf{t}^{\operatorname{Des}(T)} .
$$

Applying the vector space isomorphism from the multilinear subspace of the formal power series ring $\mathbb{Z}\left[t_{1}, t_{2}, \ldots\right]$ to the ring of quasisymmetric functions, defined by $\mathbf{t}^{J} \mapsto \mathcal{F}_{n, J}$, we get

$$
\mathcal{Q}(A)=\sum_{\lambda / \mu \vdash n} b_{\lambda / \mu} \sum_{T \in \operatorname{SYT}(\lambda / \mu)} \mathcal{F}_{n, \operatorname{Des}(T)}=\sum_{\lambda / \mu \vdash n} b_{\lambda / \mu} s_{\lambda / \mu},
$$

where the last equality uses Gessel's identity [16, Theorem 7.19.7].

Using the Littlewood-Richardson rule, which expresses skew Schur functions as nonnegative linear combinations of Schur functions [16, Eq. (A1.142)], we see that $A$ is Schur-positive.

Finally, by Equation (5), $A$ is cDes-invariant because so is the corresponding collection of SYT of the skew shapes given by the right-hand side.

\subsection{Horizontal rotations}

We conclude this section with some applications of Theorem 15 to sets of permutations that are invariant under horizontal rotation. The next result follows immediately from Theorem 15 together with Remark 14.

Theorem 22. If $A \subseteq \mathfrak{S}_{n}$ is Schur-positive and invariant under horizontal rotation, then it is $c S p$.

Another consequence is the fact that horizontal rotation closures of Schur-positive sets are cSp.

Theorem 23. For every Schur-positive set $A \subseteq \mathfrak{S}_{n-1}$, the set $A C_{n} \subseteq \mathfrak{S}_{n}$ is $c S p$.

Proof of Theorem 23. For every Schur-positive set $A \subseteq \mathfrak{S}_{n-1}$, the set $A C_{n} \subseteq \mathfrak{S}_{n}$ is Schurpositive by [10, Theorem 1.1]. Since $A C_{n}$ is invariant under horizontal rotation, Theorem 22 implies that it is cSp.

Define the cyclic descent number of $\pi$ as $\operatorname{cdes}(\pi):=|\operatorname{cDes}(\pi)|$.

Corollary 24. For every $n>k \geqslant 1$, the set

$$
C_{n, k}:=\left\{\pi \in \mathfrak{S}_{n}: \operatorname{cdes}\left(\pi^{-1}\right)=k\right\}
$$

is $c S p$.

Proof. It is shown in [9, Corollary 7.7] that $C_{n, k}$ is Schur-positive. By [9, Lemma 6.4], it is invariant under horizontal rotation. Thus, by Theorem 22, it is cSp.

A more transparent, self-contained proof of Corollary 24 will be given in Section 4.2. 


\section{Vertical versus horizontal rotations}

In this section we prove the following equidistribution result, and we discuss applications of it. Recall the definition of $D_{n-1, J}$ from Equation (6).

Theorem 25. For every $J \subseteq[n-2]$,

$$
\sum_{\pi \in C_{n} D_{n-1, J}^{-1}} \mathbf{t}^{\mathrm{cDes}(\pi)} q^{\pi^{-1}(n)}=\sum_{\pi \in D_{n-1, J}^{-1} C_{n}} \mathbf{t}^{\mathrm{cDes}(\pi)} q^{\pi^{-1}(n)} .
$$

Our proof is not bijective, but explicit bijections for the special cases when $J=\{j\}$ (singletons) and $J=[i]$ (prefixes) will be given in Sections 5 and 6 , respectively.

\subsection{Proof of Theorem 25}

We first recall some basic definitions. A composition (respectively, weak composition) of $n \geqslant 0$ is a finite sequence of positive (respectively, nonnegative) integers $\gamma=\left(\gamma_{1}, \ldots, \gamma_{t}\right)$ whose sum is $n$.

For a positive integer $n$, there is a natural bijection from subsets of $[n-1]$ to compositions of $n$. Indeed, to the subset $J=\left\{j_{1}<j_{2}<\cdots<j_{t-1}\right\} \subseteq[n-1]$ we associate the composition $\gamma=\left(\gamma_{1}, \ldots, \gamma_{t}\right)$ of $n$ defined as follows: set $j_{0}=0$ and $j_{t}=n$, and let $\gamma_{i}=j_{i}-j_{i-1}$, for $1 \leqslant i \leqslant t$. Further, define

$$
S(\gamma):=\bigsqcup_{I \subseteq J} D_{n, I}^{-1}
$$

where $\sqcup$ denotes disjoint union. In other words, $S(\gamma)$ is the set of permutations where, for each $1 \leqslant i \leqslant t$, the entries $j_{i-1}+1, j_{i-1}+2, \ldots, j_{i}$ appear from left to right in increasing order. At times we also denote this set by $S\left(\gamma_{1}, \ldots, \gamma_{t}\right)$ when consideration of the parts in $\gamma$ is required. Refining this set, let

$$
S\left(\gamma_{1}, \ldots, \gamma_{i}^{*}, \ldots, \gamma_{t}\right):=\left\{\pi \in S(\gamma) \mid \pi(n)=j_{i}\right\}
$$

and

$$
S\left(\gamma_{1}, \ldots,{ }_{*} \gamma_{i}, \ldots, \gamma_{t}\right):=\left\{\pi \in S(\gamma) \mid \pi(1)=j_{i-1}+1\right\}
$$

so that

$$
\bigsqcup_{i \in[t]} S\left(\gamma_{1}, \ldots, \gamma_{i}^{*}, \ldots, \gamma_{t}\right)=S(\gamma)=\bigsqcup_{i \in[t]} S\left(\gamma_{1}, \ldots, \gamma_{i}, \ldots, \gamma_{t}\right) .
$$

For the remainder of this section, fix a composition $\gamma=\left(\gamma_{1}, \ldots, \gamma_{t}\right)$ of $n$ and denote by $J \subseteq[n-1]$ its corresponding subset. The relevance of these definitions to the theorem at hand is the fact that

$$
\bigsqcup_{I \subseteq J} C_{n} D_{n-1, I}^{-1}=C_{n} S\left(\gamma_{1}, \ldots, \gamma_{t}^{*}\right) \quad \text { and } \bigsqcup_{I \subseteq J} D_{n-1, I}^{-1} C_{n}=S\left(\gamma_{1}, \ldots, \gamma_{t}^{*}\right) C_{n}
$$


As we shall see, it is easier to first argue in terms of such unions and then, via an application of inclusion-exclusion, conclude our desired result. To keep track of the position of the largest letter, we introduce two further refinements. Let

$$
\mathcal{V}_{\gamma}^{k}=\left\{\pi \in C_{n} S\left(\gamma_{1}, \ldots, \gamma_{t}^{*}\right): \pi(k)=n\right\},
$$

so that $\mathcal{V}_{\gamma}^{k}$ is the result of vertically rotating the permutations in $S\left(\gamma_{1}, \ldots, \gamma_{t}^{*}\right)$ until the largest value is in the $k$ th position. Likewise, define

$$
\mathcal{H}_{\gamma}^{k}=\left\{\pi \in S\left(\gamma_{1}, \ldots, \gamma_{t}^{*}\right) C_{n}: \pi(k)=n\right\},
$$

so that this set is the result of horizontally rotating the same set of permutations until the largest letter is again in the $k$ th position. We can now state our main technical lemma from which the proof of Theorem 25 follows almost immediately.

Lemma 26. For every $k \leqslant n$, there exists a cDes-preserving bijection between $\mathcal{V}_{\gamma}^{k}$ and $\mathcal{H}_{\gamma}^{k}$.

Assuming this lemma for the moment, let us prove the theorem.

Proof of Theorem 25. By Lemma 26, it follows that

$$
\sum_{\pi \in C_{n} S\left(\gamma_{1}, \ldots, \gamma_{t}^{*}\right)} \mathbf{t}^{\mathrm{cDes}(\pi)} q^{\pi^{-1}(n)}=\sum_{\pi \in S\left(\gamma_{1}, \ldots, \gamma_{t}^{*}\right) C_{n}} \mathbf{t}^{\mathrm{cDes}(\pi)} q^{\pi^{-1}(n)} .
$$

With $J \subseteq[n-1]$ defined as above, it follows from (14) and the principle of inclusionexclusion that

$$
C_{n} D_{n-1, J}^{-1}=\sum_{I \subseteq J}(-1)^{|J \backslash I|} C_{n} S\left(\gamma_{1}, \ldots, \gamma_{t}^{*}\right) .
$$

Additionally, the analogous equality involving right multiplication by $C_{n}$ also holds. Together, these facts yield the desired statement.

To prove Lemma 26 , we recall the notion of a shuffle. Let $\iota_{n}:=12 \ldots n$ denote the increasing permutation. For any nonnegative integers $a$ and $b$, define

$$
\iota_{a} \amalg \iota_{b}
$$

to be the set of permutations in $\mathfrak{S}_{a+b}$ where the letters in $[a]$ appear from left to right in increasing order, and so do the letters in $[a+b] \backslash[a]$. By definition,

$$
S\left(\gamma_{1}, \ldots, \gamma_{t}\right)=\bigsqcup_{I \subseteq J} D_{n, I}^{-1}=\iota_{\gamma_{1}} \uplus \cdots \uplus \iota_{\gamma_{t}} .
$$

The next lemma belongs to mathematical folklore, but we include a proof for the sake of completeness, as well as some examples.

Lemma 27. For every $a, b>0$, there exists a Des-preserving bijection

$$
\varphi: \iota_{a} \amalg \iota_{b} \rightarrow \iota_{b} \amalg \iota_{a} .
$$


Proof. Permutations $\pi \in \iota_{a} \amalg \iota_{b}$ can be encoded bijectively as words $w$ over a binary alphabet $\{1,2\}$ with $a$ 1s and $b 2 \mathrm{~s}$, where $w_{i}=1$ if $\pi_{i} \leqslant a$, and $w_{i}=2$ if $\pi_{i}>a$.

Given $\pi \in \iota_{a} \amalg \iota_{b}$, let $w$ its corresponding binary word. By splitting $w$ at the descents of $\pi$, which correspond to occurrences of 21 in $w$, we can write

$$
w=1^{i_{1}} 2^{j_{1}} 2\left|11^{i_{2}} 2^{j_{2}} 2\right| 1 \ldots 2 \mid 11^{i_{k}} 2^{j_{k}},
$$

with $i_{r}, j_{r} \geqslant 0$ for all $r$. Let

$$
f(w)=1^{j_{1}} 2^{i_{1}} 2\left|11^{j_{2}} 2^{i_{2}} 2\right| 1 \ldots 2 \mid 11^{j_{k}} 2^{i_{k}}
$$

and define $\varphi(\pi)$ to be the permutation in $\iota_{b} ш \iota_{a}$ encoded by $f(w)$. By construction, $\operatorname{Des}(\varphi(\pi))=\operatorname{Des}(\pi)$.

Example 28. Let $\pi=123894101156127 \in \iota_{7} \sqcup \iota_{5}$, which has $\operatorname{Des}(\pi)=\{5,8,11\}$. Encoding $\pi$ as a binary word and splitting at the descents, we get $w=11122|122| 112 \mid 1$. Applying $f$ from the second proof of Lemma 27, we get the word $f(w)=12222|112| 122 \mid 1$, which encodes the permutation $\varphi(\pi)=167892310411125 \in \iota_{5} \amalg \iota_{7}$.

It is possible to extend the construction in Lemma 27 to shuffles of $t \geqslant 2$ increasing sequences. Let $a_{1}, \ldots, a_{t}, n$ be positive integers such that $a_{1}+\cdots+a_{t}=n$. We can explicitly construct a bijection

$$
\varphi: \iota_{a_{1}} \sqcup \cdots \sqcup \iota_{a_{t}} \rightarrow \iota_{a_{t}} \amalg \cdots \sqcup \iota_{a_{1}}
$$

as follows.

For a given word $u$ over the alphabet $\{1, \ldots, t\}$ and $1 \leqslant j<t$, define $f_{j}(u)$ to be the word obtained by fixing in place all the entries of $u$ that are not equal to $j$ or $j+1$, and applying the map $f$ from the second proof of Lemma 27 to the subword of $u$ consisting of the entries $j$ and $j+1$ (ignoring the other entries).

Given $\pi \in \iota_{a_{1}} \uplus \cdots \uplus \iota_{a_{t}}$, we can encode it as a word $w$ of length $n$ over the alphabet $\{1, \ldots, t\}$ with $a_{j} j$ s for $1 \leqslant j \leqslant t$, by letting $w_{i}=j$ if $a_{1}+\cdots+a_{j-1}<\pi_{i} \leqslant a_{1}+\cdots+a_{j}$, for all $i$.

Fix a reduced decomposition of the decreasing permutation $t \ldots 21$, say $t \ldots 21=$ $s_{j_{1}} s_{j_{2}} \ldots s_{j_{r}}$ (where $r=\left(\begin{array}{l}t \\ 2\end{array}\right)$ ), and apply the map $f_{s_{j_{1}}} \circ f_{s_{j_{2}}} \circ \cdots \circ f_{s_{j_{r}}}$ to $w$. Let $\varphi(\pi)$ be the permutation in $\iota_{a_{t}} \uplus \cdots \uplus \iota_{a_{1}}$ encoded by the resulting word.

Example 29. Let $\pi=1672123891310414511 \in \iota_{5} \uplus \iota_{6} \uplus \iota_{3}$, which is encoded by the word $w=12213122321312$. Note that $\operatorname{Des}(\pi)=\{3,5,9,10,12\}$. Fixing the reduced decomposition $321=s_{1} s_{2} s_{1}$, we get

$$
\begin{aligned}
& 122|1312232| 1312 \stackrel{f_{1}}{\mapsto} \quad 122|1311232| 1312 \\
& 1221311|23| 2131\left|2 \stackrel{f_{2}}{\mapsto} 1331311\right| 23|2131| 2 \\
& 1331311232\left|1312 \stackrel{f_{1}}{\mapsto} 1332322232\right| 1312,
\end{aligned}
$$

and so $\varphi(\pi)=1101141256713821439 \in \iota_{3} \amalg \iota_{6} \amalg \iota_{5}$. 
The definition provides a "horizontal" decomposition of permutations that arise from shuffles.

Definition 30. Let $\alpha$ and $\beta$ be weak compositions, each with $t$ parts. Further, assume that $\gamma=\alpha+\beta$, where addition is componentwise. For $\rho \in S\left(\alpha_{1}, \ldots, \alpha_{t}\right)$ and $\sigma \in$ $S\left(\beta_{1}, \ldots, \beta_{t}\right)$, let

$$
\rho \circledast \sigma
$$

be the unique permutation in $S\left(\gamma_{1}, \ldots, \gamma_{t}\right)$ whose leftmost $|\alpha|$ entries are order-isomorphic to $\rho$ and whose rightmost $|\beta|$ entries are order-isomorphic to $\sigma$.

A pictorial representation of this construction when $t=3$ is given in Figure 2. In particular, if $\alpha=(2,2,0), \beta=(3,1,2)$, and we take $1342 \in S(\alpha)$ and $561423 \in S(\beta)$, then

$$
1342 \circledast 561423=16729103845 .
$$

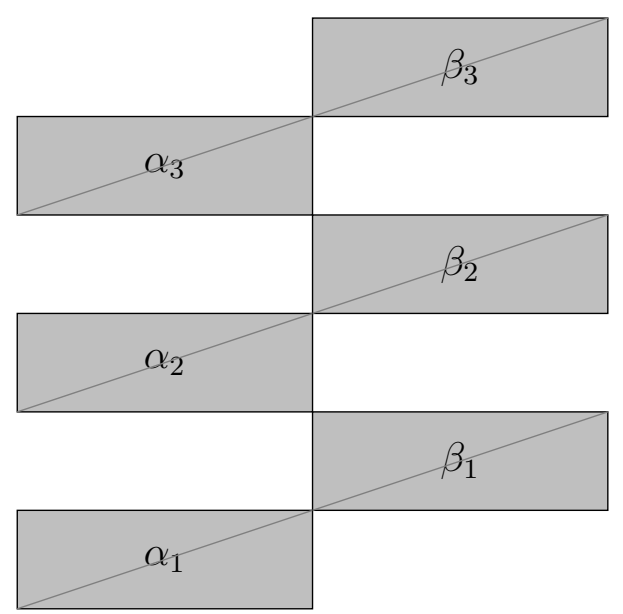

Figure 2: A visualization for $t=3$ of $\rho \circledast \sigma$ as given by Definition 30. We place (maintaining their positions) the values in $\sigma$ corresponding to $\iota_{\alpha_{i}}$ in the box labeled $\alpha_{i}$, and similarly for $\rho$.

An immediate consequence of this construction is that, for every fixed $k \leqslant n$, we have

$$
S\left(\gamma_{1}, \ldots, \gamma_{t}\right)=\bigsqcup_{(\alpha, \beta) \in \mathcal{C}_{k, n-k}^{\gamma}} S\left(\alpha_{1}, \ldots, \alpha_{t}\right) \circledast S\left(\beta_{1}, \ldots, \beta_{t}\right),
$$

where $\mathcal{C}_{k, n-k}^{\gamma}$ denotes the set of pairs of weak compositions $\alpha$ of $k$ and $\beta$ of $n-k$, each with $t$ parts, such that $\alpha+\beta=\gamma$.

For our next lemma, recall that $c_{n}=(1,2, \ldots, n)$, and that $\mathcal{V}_{\gamma}^{k}$ was defined in Equation (15).

Lemma 31. For every $1 \leqslant k \leqslant n$,

$$
\mathcal{V}_{\gamma}^{k} c_{n}^{k}=\bigsqcup_{(\alpha, \beta) \in \mathcal{C}_{k, n-k}^{\gamma}} \bigsqcup_{i \in[t]} S\left(\beta_{i}, \ldots, \beta_{t}^{*}, \beta_{1} \ldots, \beta_{i-1}\right) \circledast S\left(\alpha_{i+1}, \ldots, \alpha_{t}, \alpha_{1}, \ldots, \alpha_{i}^{*}\right) .
$$


Proof. Observe that

$$
S\left(\gamma_{1}, \ldots, \gamma_{t}^{*}\right)=\bigsqcup_{(\alpha, \beta) \in \mathcal{C}_{k, n-k}^{\gamma}} S\left(\alpha_{1}, \ldots, \alpha_{t}\right) \circledast S\left(\beta_{1}, \ldots, \beta_{t}^{*}\right) .
$$

Refining this set by the value in the $k$ th position, we get

$$
S\left(\gamma_{1}, \ldots, \gamma_{t}^{*}\right)=\bigsqcup_{(\alpha, \beta) \in \mathcal{C}_{k, n-k}^{\gamma}} \bigsqcup_{i \in[t]} S\left(\alpha_{1}, \ldots, \alpha_{i}^{*}, \ldots, \alpha_{t}\right) \circledast S\left(\beta_{1}, \ldots, \beta_{t}^{*}\right) .
$$

To obtain our claim, vertically rotate each permutation in (17) so that the value in the $k$ th position is largest, and then horizontally rotate the resulting permutation $k$ positions to the left.

The decomposition in the previous lemma motivates the next result.

Lemma 32. There is a cDes-preserving bijection between

$$
\bigsqcup_{i \in[t]} S\left(\beta_{i}, \ldots, \beta_{t}^{*}, \beta_{1}, \ldots, \beta_{i-1}\right) \circledast S\left(\alpha_{i+1}, \ldots, \alpha_{t}, \alpha_{1}, \ldots, \alpha_{i}^{*}\right)
$$

and

$$
S\left(\beta_{t}-1, \beta_{t-1}, \ldots, \beta_{1}\right) \circledast S\left(\alpha_{t}, \alpha_{t-1}, \ldots, \alpha_{2},\left(\alpha_{1}+1\right)^{*}\right) .
$$

Proof. Since all the permutations $\pi$ in the sets (18) and (19) are such that $\pi_{n}=n$, it suffices to prove the existence of a Des-preserving bijection between

$$
\mathcal{U}:=\bigsqcup_{i \in[t]} S\left(\beta_{i}, \ldots, \beta_{t}^{*}, \beta_{1}, \ldots, \beta_{i-1}\right) \circledast S\left(\alpha_{i+1}, \ldots, \alpha_{t}, \alpha_{1}, \ldots, \alpha_{i}-1\right)
$$

and

$$
\mathcal{W}:=S\left(\beta_{t}-1, \beta_{t-1}, \ldots, \beta_{1}\right) \circledast S\left(\alpha_{t}, \ldots, \alpha_{1}\right),
$$

i.e., the sets obtained from (18) and (19) by deleting this largest final value. We use the notation $\mathcal{A} \sim \mathcal{B}$ to denote that there exists a Des-preserving bijection between the sets $\mathcal{A}$ and $\mathcal{B}$. To show that $\mathcal{U} \sim \mathcal{W}$, we will construct a sequence of Des-preserving bijections.

Using Lemma 27 repeatedly, we can reorder $\beta_{1}, \ldots, \beta_{i-1}$ and, separately, reorder $\beta_{i}, \ldots, \beta_{t}^{*}$ to obtain

$$
S\left(\beta_{i}, \ldots, \beta_{t}^{*}, \beta_{1}, \ldots, \beta_{i-1}\right) \sim S\left(\beta_{t}-1, \beta_{t-1}, \ldots,\left(\beta_{i}+1\right)^{*}, \ldots, \beta_{1}\right) .
$$

As the permutations on both sides of Equation (20) all have the same value $\beta_{i}+\cdots+\beta_{t}$ in the last position, it follows that

$$
\mathcal{U} \sim \bigsqcup_{i \in[t]} S\left(\beta_{t}-1, \beta_{t-1}, \ldots,\left(\beta_{i}+1\right)^{*}, \ldots, \beta_{1}\right) \circledast S\left(\alpha_{i+1}, \ldots, \alpha_{t}, \alpha_{1}, \ldots, \alpha_{i}-1\right) .
$$


When $i=1$, the set inside the disjoint union becomes

$$
\begin{aligned}
& S\left(\beta_{t}-1, \beta_{t-1}, \ldots,\left(\beta_{1}+1\right)^{*}\right) \circledast S\left(\alpha_{2}, \ldots, \alpha_{t}, \alpha_{1}-1\right) \\
& \sim S\left(\beta_{t}-1, \beta_{t-1}, \ldots, \beta_{1}\right) \circledast S\left(\alpha_{2}, \ldots, \alpha_{t},{ }_{*} \alpha_{1}\right) \\
& \sim S\left(\beta_{t}-1, \beta_{t-1}, \ldots, \beta_{1}\right) \circledast S\left(\alpha_{t}, \ldots, \alpha_{2},{ }_{*} \alpha_{1}\right)
\end{aligned}
$$

and when $i \neq 1$, we have

$$
\begin{aligned}
& \bigsqcup_{i \neq 1} S\left(\beta_{t}-1, \beta_{t-1}, \ldots, \beta_{1}\right) \circledast S\left(\alpha_{i+1}, \ldots, \alpha_{t}, *\left(\alpha_{1}+1\right), \ldots, \alpha_{i}-1\right) \\
& \sim \bigsqcup_{i \neq 1} S\left(\beta_{t}-1, \beta_{t-1}, \ldots, \beta_{1}\right) \circledast S\left(\alpha_{t}, \ldots,{ }_{*} \alpha_{i}, \ldots, \alpha_{1}\right),
\end{aligned}
$$

where the equivalence follows by interchanging the order of the $\beta$ 's just as we did above with the $\alpha$ 's. Summing over $i \in[t]$ shows that there exists a Des-preserving bijection between $\mathcal{U}$ and $\mathcal{W}$, proving our lemma.

Armed with these technical lemmas, we prove Lemma 26.

Proof of Lemma 26. For any two sets $\mathcal{A}, \mathcal{B} \subseteq \mathfrak{S}_{n}$, there exists a cDes-preserving bijection between $\mathcal{A}$ and $\mathcal{B}$ if and only if there is one between $\mathcal{A} c_{n}^{k}$ and $\mathcal{B} c_{n}^{k}$. Thus, to prove the lemma, it suffices to show that such a bijection exists between $\mathcal{H}_{\gamma}^{k} c_{n}^{k}$ and $\mathcal{V}_{\gamma}^{k} c_{n}^{k}$. By the definitions involved, we have

$$
\mathcal{H}_{\gamma}^{k} c_{n}^{k}=S\left(\gamma_{1}, \ldots, \gamma_{t}^{*}\right) .
$$

On the other hand, by Lemmas 31 and 32, there is a cDes-preserving bijection between $\mathcal{V}_{\gamma}^{k} c_{n}^{k}$ and

$\bigsqcup_{(\alpha, \beta) \in \mathcal{C}_{k, n-k}^{\gamma}} S\left(\beta_{t}-1, \beta_{t-1}, \ldots, \beta_{1}\right) \circledast S\left(\alpha_{t}, \ldots,\left(\alpha_{1}+1\right)^{*}\right)=S\left(\gamma_{t}-1, \gamma_{t-1}, \ldots, \gamma_{2},\left(\gamma_{1}+1\right)^{*}\right)$.

Applying Lemma 27 to the right-hand side and noting that all permutations end with the largest letter $n$, we get a cDes-preserving bijection between this set and $S\left(\gamma_{1}, \ldots, \gamma_{t}^{*}\right)$. This completes our proof.

\subsection{Consequences of Theorem 25}

The following is one of the main applications of the theorem.

Theorem 33. For every $J \subseteq[n-2]$, the vertically rotated inverse descent class $C_{n} D_{n-1, J}^{-1}$ in $\mathfrak{S}_{n}$ is $c S p$.

Proof. As shown in [11] (see also Table 1), inverse descent classes are Schur-positive. Hence, by Theorem 23, for every $J \subseteq[n-2]$, the horizontally rotated inverse descent class $D_{n-1, J}^{-1} C_{n}$ is cSp. Finally, by Theorem 25, the distribution of cDes on $C_{n} D_{n-1, J}^{-1}$ is the same as on $D_{n-1, J}^{-1} C_{n}$, completing the proof. 
Next we apply Theorem 33 to give a self-contained proof of Corollary 24, which states that the set of permutations with a given inverse cyclic descent number is cSp.

Second proof of Corollary 24. Note that

$$
\left\{\pi \in \mathfrak{S}_{n}: \operatorname{cdes}(\pi)=k\right\}=\bigsqcup_{\substack{J \subseteq[n-2] \\|J|=k-1}} D_{n-1, J} C_{n} .
$$

Thus

$$
C_{n, k}=\left\{\pi \in \mathfrak{S}_{n}: \operatorname{cdes}\left(\pi^{-1}\right)=k\right\}=\bigsqcup_{\substack{J \subseteq[n-2] \\|J|=k-1}} C_{n} D_{n-1, J}^{-1} .
$$

Theorem 33 completes the proof.

The set $C_{n, k}$ had been shown to be Schur-positive in [9, Corollary 7.7]. A significantly stronger version of this result is conjectured in [2, Conjecture 7.1]. Originally formulated in the language of cyclic quasi-symmetric functions, cyclic compositions and cyclic permutations, this conjecture equivalent to the statement that, for every $\emptyset \subsetneq J \subsetneq[n]$, the set of permutations in $\mathfrak{S}_{n}$ whose inverse has cyclic descent set of the form $i+J$ for some $i$ (with addition modulo $n$ as usual) is Schur-positive. The following consequence of Theorem 33 provides an affirmative solution to this conjecture.

Corollary 34. For every $\emptyset \subsetneq J \subsetneq[n]$, the set

$$
\left\{\pi \in \mathfrak{S}_{n}: \operatorname{cDes}\left(\pi^{-1}\right)=i+J \text { for some } i\right\}
$$

is cSp. In particular, it is Schur-positive.

Proof. For $j \in J$, let $\Delta_{j}:=D_{n-1,-j+(J \backslash\{j\})}$, viewed as a subset of $\mathfrak{S}_{n}$, and let $A$ denote the set in Equation (21).

Given $\pi \in \mathfrak{S}_{n}$, we have that $\operatorname{cDes}(\pi)=J$ if and only if there exists $j \in J$ such that $j-1 \notin J$ and $\pi=\sigma c_{n}^{j}$ for some $\sigma \in \Delta_{j}$. Indeed, the 'if' direction is clear, and the other is obtained by taking $j=\pi^{-1}(n)$. It follows that $\operatorname{cDes}(\pi)=i+J$ if and only if there exists $j \in J$ such that $j-1 \notin J$ and $\pi=\sigma c_{n}^{j+i}$ for some $\sigma \in \Delta_{j}$. Letting $i$ vary, we get

$$
A^{-1}=\bigcup_{\substack{j \in J, j-1 \notin J}} \Delta_{j} C_{n}
$$

Any two sets in this union are either equal or disjoint. Specifically, $\Delta_{j_{1}} C_{n} \cap \Delta_{j_{2}} C_{n}=\emptyset$ unless $-j_{1}+J=-j_{2}+J$, in which case $\Delta_{j_{1}} C_{n}=\Delta_{j_{2}} C_{n}$. It follows that we can express $A^{-1}$ as a disjoint union of sets $\Delta_{j} C_{n}$ for $j$ in some subset $J^{\prime} \subseteq J$. Taking inverses, we get

$$
A=\bigsqcup_{j \in J^{\prime}} C_{n} \Delta_{j}^{-1}
$$

which is cSp by Theorem 33, and thus Schur-positive by Theorem 15 . 
Example 35. Let $n=4$ and $J=\{2,4\}$. Then $-2+J \backslash\{2\}=-4+J \backslash\{4\}=\{2\}$, so

$$
\left\{\pi \in \mathfrak{S}_{4}: \operatorname{cDes}\left(\pi^{-1}\right)=\{2,4\} \text { or }\{1,3\}\right\}=C_{4} D_{3,\{2\}}^{-1} .
$$

Example 36. Let $n=6$ and $J=\{1,3,5\}$. Then $-1+J \backslash\{1\}=-3+J \backslash\{3\}=$ $-5+J \backslash\{5\}=\{2,4\}$, so

$$
\left\{\pi \in \mathfrak{S}_{6}: \operatorname{cDes}\left(\pi^{-1}\right)=\{1,3,5\} \text { or }\{2,4,6\}\right\}=C_{6} D_{5,\{2,4\}}^{-1} .
$$

Example 37. Let $n=5$ and $J=\{1,2,4\}$. Then $-1+J \backslash\{1\}=\{1,3\}$ and $-4+J \backslash\{4\}=$ $\{2,3\}$, so

$$
\left\{\pi \in \mathfrak{S}_{5}: \operatorname{cDes}\left(\pi^{-1}\right)=i+\{1,2,4\} \text { for some } i\right\}=C_{5} D_{4,\{1,3\}}^{-1} \sqcup C_{5} D_{4,\{2,3\}}^{-1} \text {. }
$$

Our last application of Theorem 25 is an affirmative solution to [9, Conjecture 10.2].

Corollary 38 ([9, Conjecture 10.2]). $\quad$ 1. For every $J \subseteq[n-2]$, the distribution of Des over the vertically rotated inverse descent class $C_{n} D_{n-1, J}^{-1}$ is the same as over $D_{n-1, J}^{-1} C_{n}$.

2. The set $C_{n} D_{n-1, J}^{-1}$ is Schur-positive.

Proof. To prove part 1 , let $t_{n}=q=1$ in Theorem 25. For part 2, we know that $C_{n} D_{n-1, J}^{-1}$ is cSp by Theorem 33, and thus it is Schur-positive by Theorem 15 .

\section{$5 \quad$ An explicit bijection for shuffles of two increasing sequences}

In this section and the next one we describe explicit bijections proving Theorem 25 in two special cases. This section deals with the case that $|J|=1$, and Section 6 will deal with the case $J=[i]$.

In the rest of this section, suppose that $J$ contains one element, that is, $J=\{j\}$. In this case, $D_{n-1, J}^{-1}$ consists of shuffles of two increasing sequences of fixed length. Specifically, $D_{n-1, J}^{-1}=S\left(j,(n-j)^{*}\right) \backslash\{12 \ldots n\}$. We give an explicit bijection

$$
\Psi: C_{n} D_{n-1,\{j\}}^{-1} \rightarrow D_{n-1,\{j\}}^{-1} C_{n}
$$

that preserves cDes and the position of $n$. This bijection is obtained by analyzing the proof of Theorem 25, and using the map $\varphi$ in the proof of Lemma 27 to make the bijection explicit.

For a word $w$ over the alphabet $\{1,2\}$, recall from the proof of Lemma 27 that $f(w)$ is obtained by keeping the descents 21 untouched, and changing each consecutive block of letters of $w$ that is not part of a 21 , which must be of the form $1^{r} 2^{s}$, into $1^{s} 2^{r}$. Additionally, for $p<q$, define $f_{[p, q]}(w)$ to be the word obtained by applying the above operation $f$ to the factor $w_{p} w_{p+1} \ldots w_{q}$, and keeping the other entries of $w$ unchanged. 
Next we describe how to obtain $\Psi(\pi)$ for given $\pi \in C_{n} D_{n-1,\{j\}}^{-1}$. Let $k=\pi^{-1}(n)$ be the position of $n$. If $k=n$, we simply define $\Psi(\pi)=\pi$. Suppose now that $k \neq n$. Rotate $\pi$ horizontally $k$ positions to the left, so that its rightmost entry becomes $n$. The resulting permutation $\pi c_{n}^{k}$ is a shuffle of two increasing sequences, so it can be encoded as a word $w$ over $\{1,2\}$ by placing $1 \mathrm{~s}$ in the positions corresponding to the lower increasing sequence, and $2 \mathrm{~s}$ elsewhere. If $w_{n-k}=1$, let $w^{\prime}=f_{[n-k+1, n-1]}(w)$, else (that is, if $w_{n-k}=2$ ) let $w^{\prime}=f_{[1, n-k-1]}(w)$. Let $w^{\prime \prime}=f_{[1, n-1]}\left(w^{\prime}\right)$, and let $\sigma$ be the permutation in $D_{n-1,\{j\}}^{-1}$ (viewed as a subset of $\mathfrak{S}_{n}$ ) encoded by $w^{\prime \prime}$. Let $\Psi(\pi)=\sigma c_{n}^{-k}$, that is, the permutation obtained by rotating $\sigma$ horizontally $k$ positions to the right. A schematic description of this construction is given in Figure 3.

Example 39. Let $n=16$ and $J=\{7\}$. Let

$$
\pi=13614157161891023411125 \in C_{n} D_{n-1, J}^{-1},
$$

which is a vertical rotation of

$$
81910211123451314156716 \in D_{n-1, J}^{-1}
$$

(viewed as a subset of $\mathfrak{S}_{n}$ ). Since $k=\pi^{-1}(16)=6$, we first compute

$$
\pi c_{n}^{6}=18910234111251361415716,
$$

which is encoded by the word $w=1222111221212212$. Since $w_{10}=1$, we define

$$
w^{\prime}=f_{[11,15]}(1222111221212212)=1222111221211212,
$$

and

$$
w^{\prime \prime}=f_{[1,15]}(1222111221211212)=1122112221212212 .
$$

This word encodes the permutation $\sigma=12893410111251361415716$, and so $\Psi(\pi)=$ $\sigma c_{n}^{-6}=13614157161289341011125$.

\section{Arc permutations}

In this section we give an explicit bijective proof of Theorem 25 in the case $J=[i]$. Unlike the bijection $\Psi$ from Section 5, this bijection does not follow from our proof of Theorem 25. We will see that the theorem, in this special case, becomes a statement about arc permutations.

Definition 40. A permutation $\pi \in \mathfrak{S}_{n}$ is an arc permutation if, for every $1 \leqslant j \leqslant n$, the first $j$ letters in $\pi$ form an interval in $\mathbb{Z}_{n}$. Denote by $\mathcal{A}_{n}$ the set of arc permutations in $\mathfrak{S}_{n}$. 


$$
D_{n-1,\{j\}}^{-1}
$$

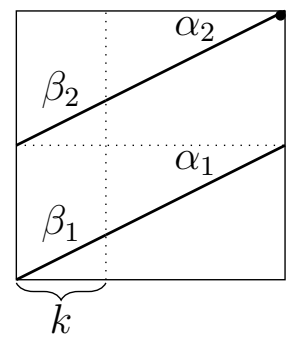
$C_{n} D_{n-1,\{j\}}^{-1}$ with $\pi(k)=n$
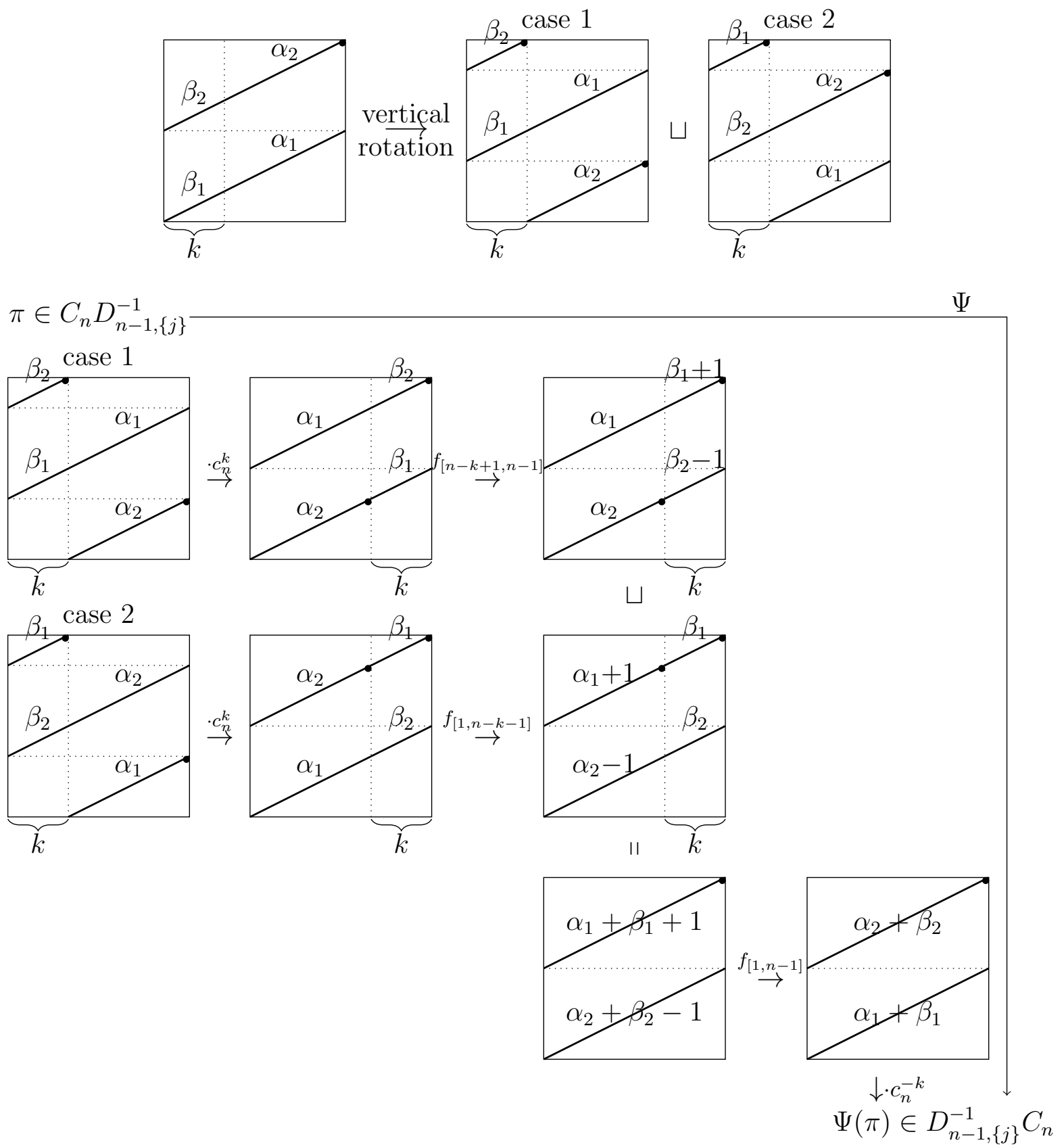

Figure 3: A schematic description of the map $\Psi$. Permutations $\pi \in C_{n} D_{n-1,\{j\}}^{-1}$ (obtained by vertically rotating $\left.D_{n-1,\{j\}}^{-1}\right)$ with $\pi(k)=n$ fall into two cases. Here $\alpha_{1}+\beta_{1}=j$, $\alpha_{2}+\beta_{2}=n-j$, and $\beta_{1}+\beta_{2}=k$. 
For example, $12543 \in \mathcal{A}_{5}$, but $125436 \notin \mathcal{A}_{6}$, since $\{1,2,5\}$ is an interval in $\mathbb{Z}_{5}$ but not in $\mathbb{Z}_{6}$.

Arc permutations were introduced in the study of flip graphs of polygon triangulations. It was shown in [8] that they can be characterized as those avoiding the eight patterns $\sigma \in \mathfrak{S}_{4}$ with $|\sigma(1)-\sigma(2)|=2$, that is,

$$
\mathcal{A}_{n}=\mathfrak{S}_{n}(1324,1342,2413,2431,3124,3142,4213,4231) .
$$

Other combinatorial properties of these permutations, such as their descent set distribution, are studied in [8]. In particular, it follows from [8, Theorem 5] that $\mathcal{A}_{n}$ is a Schur-positive set. Notice that while $c_{n} \mathcal{A}_{n}=\mathcal{A}_{n}$ for every $n$, we have that $\mathcal{A}_{n} c_{n} \neq \mathcal{A}_{n}$ for $n>3$. In other words, $\mathcal{A}_{n}$ is invariant under vertical rotation but not under horizontal rotation, and thus Theorem 22 does not apply. However, we will show that it is possible to express arc permutations in terms of vertical rotations of inverse descent classes.

\subsection{Permutation classes and grids}

Definition 41. A sequence of integers $a_{1}, \ldots, a_{n}$ is

- left-unimodal if it is a union of an increasing subsequence and a decreasing subsequence, which intersect at the first letter $a_{1}$;

- right-unimodal if the sequence $a_{n}, a_{n-1}, \ldots, a_{1}$ is left-unimodal.

We say that a permutation $\pi \in \mathfrak{S}_{n}$ has one of the above properties if the sequence $\pi(1), \pi(2), \ldots, \pi(n)$ does. Denote the sets of left-unimodal and right-unimodal permutations in $\mathfrak{S}_{n}$ by $\mathcal{L}_{n}$ and $\mathcal{R}_{n}$, respectively. Note that for every $n \geqslant 1, \pi \in \mathcal{L}_{n}$ (respectively, $\pi \in \mathcal{R}_{n}$ ) if and only if, for every $1 \leqslant j \leqslant n$, the first (respectively, last) $j$ letters in $\pi$ form an interval in $\mathbb{Z}$. These sets can be described in terms of pattern avoidance as $\mathcal{L}_{n}=\mathfrak{S}_{n}(132,312)$ and $\mathcal{R}_{n}=\mathfrak{S}_{n}(231,213)$, respectively.

Arc permutations are obtained as vertical rotations of left-unimodal permutations, that is, $\mathcal{A}_{n}=C_{n} \mathcal{L}_{n-1}$. Using that

$$
\mathcal{L}_{n-1}=\bigsqcup_{i=0}^{n-2} D_{n-1,[i]}^{-1},
$$

we can express $\mathcal{A}_{n}$ as a disjoint union of vertically rotated inverse descent classes:

$$
\mathcal{A}_{n}=\bigsqcup_{i=0}^{n-2} C_{n} D_{n-1,[i]}^{-1} .
$$

The next result follows now from Theorem 33 .

Corollary 42. The set $\mathcal{A}_{n}$ is cSp. 
Next we recall the notion of geometric grid classes from [5], which will be useful for our bijection in Subsection 6.2.

Definition 43. A geometric grid class consists of those permutations that can be drawn on a specified set of line segments of slope \pm 1 , arranged according to the positions of the nonzero entries of a matrix $M$ with entries in $\{0,+1,-1\}$. Specifically, a permutation $\pi \in \mathfrak{S}_{n}$ can be drawn on these line segments if $n$ points can be placed on them so that the $i$ th point from the left is the $\pi(i)$ th point from the bottom, for all $1 \leqslant i \leqslant n$.

Example 44. Left-unimodal and right-unimodal permutations are those in the geometric grid classes of the matrices

$$
M=\left(\begin{array}{c}
+1 \\
-1
\end{array}\right), M=\left(\begin{array}{c}
-1 \\
+1
\end{array}\right)
$$

respectively. A drawing of a left-unimodal permutation on the corresponding grid is shown in Figure 4.

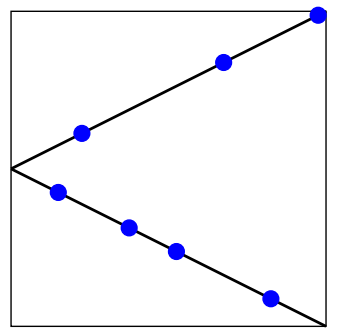

Figure 4: A drawing of the permutation 4532617 on the grid for left-unimodal permutations.

Being vertical rotations of left-unimodal permutations, arc permutations are precisely those that can be drawn on one of the grids in Figure 5, which are obtained by vertically rotating the grid in Figure 4. This pictorial grid description implies the following property.
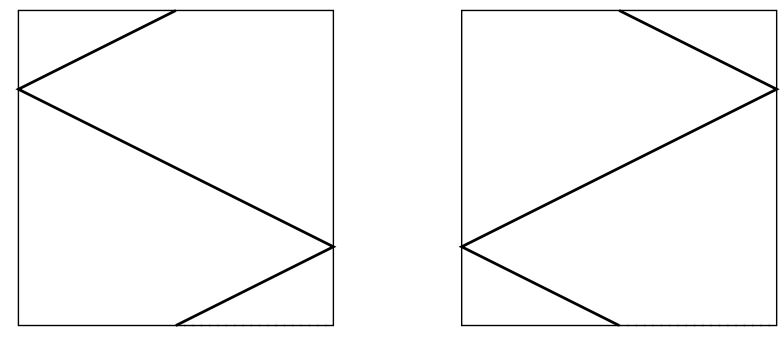

Figure 5: Grids for arc permutations.

Observation 45. A permutation $\pi \in \mathfrak{S}_{n}$ with $\pi(j)=n$ is an arc permutation if and only if one of the following holds: 
- the prefix $\pi(1), \ldots, \pi(j-1)$ is a left-unimodal sequence on $[j]$, and the suffix $\pi(j+$ $1), \ldots, \pi(n)$ is a right-unimodal sequence on $[n-1] \backslash[j]$, or

- the prefix $\pi(1), \ldots, \pi(j-1)$ is a left-unimodal sequence on $[n-1] \backslash[n-j]$, and the suffix $\pi(j+1), \ldots, \pi(n)$ is a right-unimodal sequence on $[n-j]$.

\subsection{A cDes-preserving bijection}

Next we give a bijection between $D_{n-1,[i]}^{-1} C_{n}$ and $C_{n} D_{n-1,[i]}^{-1}$ that preserves the cyclic descent set. By allowing to $i$ range between 0 and $n-2$ and using Equations (22) and (23), we obtain a bijection between $\mathcal{L}_{n-1} C_{n}$ and $\mathcal{A}_{n}$. In fact, we will describe the bijection in this setting.

A key fact used in the construction below is that the descent set map Des is a bijection between $\mathcal{L}_{n-1}$ and the power set $2^{[n-2]}$.

Definition 46. Let $\phi: \mathcal{L}_{n-1} C_{n} \longrightarrow \mathcal{A}_{n}$ be the following map. Given $\pi \in \mathcal{L}_{n-1} C_{n}$, let $j=\pi^{-1}(n)$ be the position of the letter $n$ in $\pi$, and construct $\phi \pi$ as follows:

- Let $(\phi \pi)(j)=n$.

- If $n \in \operatorname{cDes}(\pi)$, then the set of the leftmost $j-1$ entries in $\phi \pi$ is equal to $[j-1]$; otherwise, it is equal to $[n-1] \backslash[n-j]$.

- The order of the first $j-1$ entries in $\phi \pi$ is given by the only left-unimodal permutation having descent set $\operatorname{Des}(\pi) \cap[j-2]$.

- The order of the last $n-j$ entries in $\phi \pi$ is given by the only right-unimodal permutation having descent set $\{i-j: i \in \operatorname{Des}(\pi), i>j\}$.

Example 47. Take $43567281 \in \mathcal{L}_{8}$ and $\pi=672819435 \in \mathcal{L}_{8} C_{9}$. Then $j=\pi^{-1}(9)=6$, so we set $(\phi \pi)(6)=9$. Since $9 \notin \operatorname{cDes}(\pi)$, the first five entries in $\phi \pi$ are [8] \[3]= $\{4,5,6,7,8\}$, and the last three entries are $\{1,2,3\}$. The order of the first five entries is given by the only left-unimodal permutation of $\{4,5,6,7,8\}$ with descent set $\operatorname{Des}(\pi) \cap$ $[4]=\{2,4\}$, namely 67584 . The order of the last three entries is given by the only right-unimodal permutation on $\{1,2,3\}$ with descent set $\{1\}$, namely 312. Altogether, $\phi \pi=675849312 \in \mathcal{A}_{9}$. As expected, $\operatorname{cDes}(\phi \pi)=\{2,4,6,7\}=\operatorname{cDes}(\pi)$.

A pictorial description of the map $\phi$ is presented in Figure 6, with a concrete example in Figure 7. For $\pi \in \mathcal{L}_{n-1} C_{n}$ with $\pi(j)=n$, the two cases correspond to $n \in \operatorname{cDes}(\pi)$ and $n \notin \mathrm{cDes}(\pi)$, or equivalently, to $\pi(1)<\pi(j+1)$ and $\pi(1)>\pi(j+1)$, respectively. Note that when $j=n$, we have $\phi \pi=\pi$.

Lemma 48. The map $\phi$ is a cDes-preserving bijection between $\mathcal{L}_{n-1} C_{n}$ and $\mathcal{A}_{n}$, satisfying $\pi(n)=(\phi \pi)(n)$. In particular,

$$
\sum_{\pi \in \mathcal{A}_{n}} \mathbf{t}^{\mathrm{cDes}(\pi)} q^{\pi^{-1}(n)}=\sum_{\pi \in \mathcal{L}_{n-1} C_{n}} \mathbf{t}^{\mathrm{cDes}(\pi)} q^{\pi^{-1}(n)} .
$$



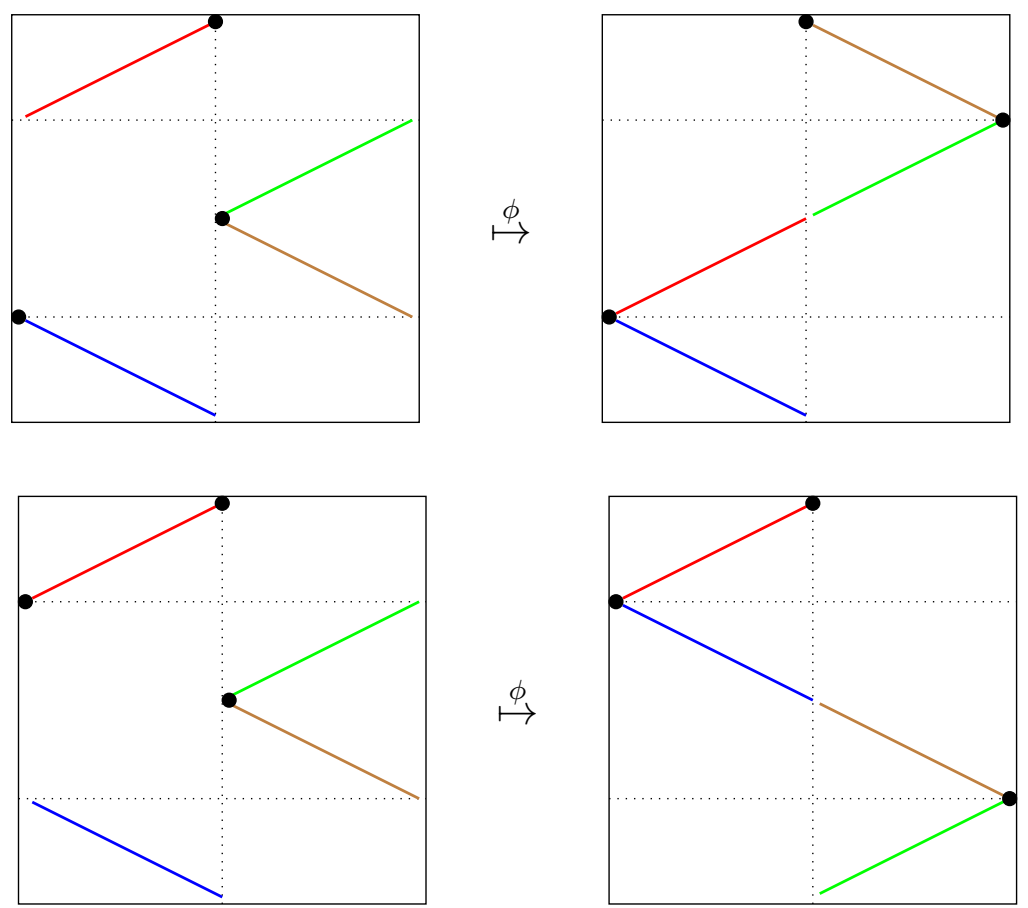

Figure 6: The cDes-preserving bijection $\phi: \mathcal{L}_{n-1} C_{n} \longrightarrow \mathcal{A}_{n}$.

Proof. By construction, $\operatorname{cDes}(\phi \pi)=\operatorname{cDes}(\pi)$, and by Observation $45, \phi \pi \in \mathcal{A}_{n}$. To prove that $\phi$ is a bijection, it suffices to describe its inverse map. Define $\psi: \mathcal{A}_{n} \longrightarrow \mathcal{L}_{n-1} C_{n}$ as follows. Given $\sigma \in \mathcal{A}_{n}$, let $j=\sigma^{-1}(n)$ be the position of $n$.

1. Let $\hat{\sigma}=\sigma c_{n}^{-j}$. Notice that $\hat{\sigma}(n)=n$ and $\operatorname{cDes}(\hat{\sigma})=-j+\operatorname{cDes}(\sigma) \bmod n$.

2. Let $\operatorname{des}(\hat{\sigma})=|\operatorname{Des}(\hat{\sigma})|$. Create a permutation $\bar{\sigma}$ by placing the decreasing sequence $\operatorname{des}(\hat{\sigma}), \ldots, 2,1$ in positions $1+\operatorname{Des}(\hat{\sigma})=\{i+1: \hat{\sigma}(i)>\hat{\sigma}(i+1)\}$, and by placing the increasing sequence $1+\operatorname{des}(\hat{\sigma}), \ldots, n-1, n$ in the remaining positions. Notice that $\bar{\sigma}$ may be identified, by ignoring $\bar{\sigma}(n)=n$, with a permutation in $\mathcal{L}_{n-1}$.

3. Let $\psi \sigma=\bar{\sigma} c^{j}$.

It is easy to verify that $\psi$ and $\phi$ are inverses of each other.

Example 49. Let $\sigma=675849312 \in \mathcal{A}_{9}$ be the permutation from Example 47. Then $j=6, \hat{\sigma}=312675849$, and $-6+\mathrm{cDes}(\pi)=-6+\{2,4,6,7\}=\{1,5,7,9\}=\operatorname{cDes}(\hat{\pi})$, with addition modulo 9 . Since $\operatorname{Des}(\hat{\sigma})=\{1,5,7\}$, we place the decreasing sequence $3,2,1$ in positions $1+\operatorname{Des}(\hat{\sigma})=\{2,6,8\}$, obtaining $* 3 * * * 2 * 1 *$, and an increasing sequence in the remaining positions, obtaining $\bar{\sigma}=435672819 \in \mathcal{L}_{8} \subseteq \mathfrak{S}_{9}$. Finally, $\psi \sigma=\bar{\sigma} c^{j}=$ 6728194356. 

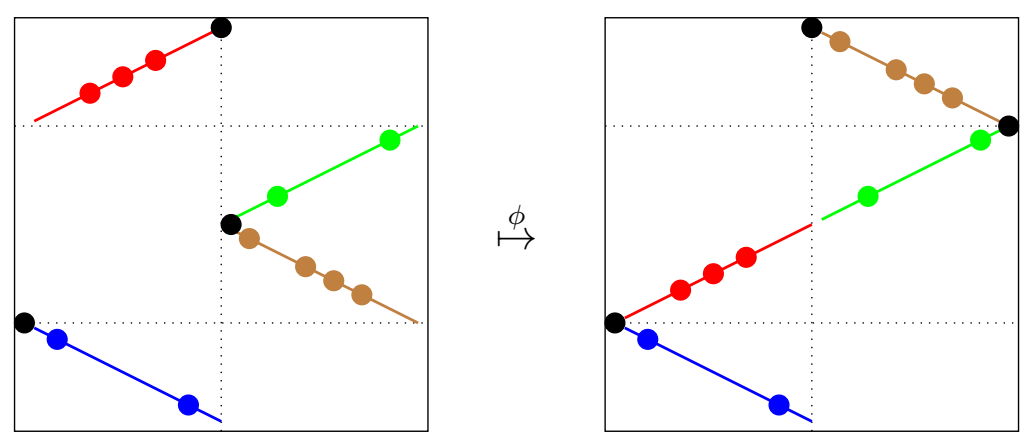

Figure 7: Example: $\phi(3211121311487965410)=3245611413712111089$.

\subsection{From arc permutations to SYT}

Next we give a bijective proof of Corollary 42. The proof, which relies on the bijection from Section 6.2, provides an explicit set of SYT on which cDes has the same distribution as it has on arc permutations (as in Definition 4).

Theorem 50. For every $n \geqslant 1$,

$$
\sum_{\pi \in \mathcal{A}_{n}} \mathbf{t}^{\mathrm{cDes}(\pi)}=\sum_{k=0}^{n-2} \sum_{T \in \operatorname{SYT}\left(\left(n-k-1,1^{k}\right) \oplus(1)\right)} \mathbf{t}^{\mathrm{cDes}(T)} .
$$

Proof. Using Equation (23), it suffices to show that for every $0 \leqslant k<n-1$,

$$
\sum_{\pi \in C_{n} D_{n-1,[k]}^{-1}} \mathbf{t}^{\mathrm{cDes}(\pi)}=\sum_{\pi \in D_{n-1,[k]}^{-1} C_{n}} \mathbf{t}^{\mathrm{cDes}(\pi)}=\sum_{T \in \operatorname{SYT}\left(\left(n-k-1,1^{k}\right) \oplus(1)\right)} \mathbf{t}^{\mathrm{cDes}(T)} .
$$

The left equality follows from Theorem 25. To prove the right equality, we construct a cDes-preserving bijection $f$ between $D_{n-1,[k]}^{-1} C_{n}$ and $\operatorname{SYT}\left(\left(n-k-1,1^{k}\right) \oplus(1)\right)$.

Given $\sigma \in D_{n-1,[k]}^{-1} C_{n}$, write $\sigma=\tau c_{n}^{-j}$, where $\tau \in D_{n-1,[k]}^{-1} \subseteq \mathfrak{S}_{n}$ and $0 \leqslant j<n$. Let $f(\tau)$ be the SYT of shape $\left(n-k-1,1^{k}\right) \oplus(1)$ having entry $n$ in the northeast component, and whose first column consists of the entry 1 and the elements in the set $1+\operatorname{Des}(\tau)$. Then let

$$
f(\sigma)=j+f(\tau),
$$

with addition defined as in Example 9. Finally, apply the definition of cDes in Equation (7) to verify that $f$ is a cDes-preserving bijection.

Composing the above bijection $f$ with the bijection $\phi^{-1}: \mathcal{A}_{n} \rightarrow \mathcal{L}_{n-1} C_{n}$ from Definition 46 , we obtain a cDes-preserving bijection from $\mathcal{A}_{n}$ to the set $\bigsqcup_{k=0}^{n-2} \operatorname{SYT}((n-k-$ $\left.\left.1,1^{k}\right) \oplus(1)\right)$. An example is shown in Figure 8. 

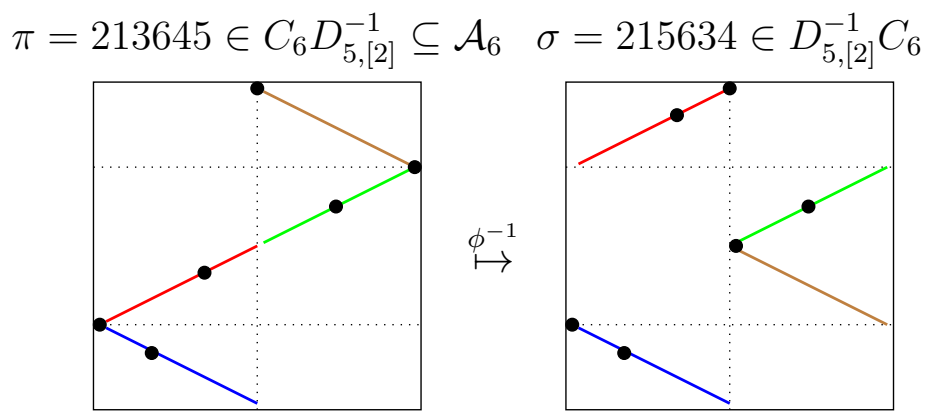

$f(\sigma) \in \operatorname{SYT}\left(\left(3,1^{2}\right) \oplus(1)\right)$

$I \cdot c_{6}^{4}$
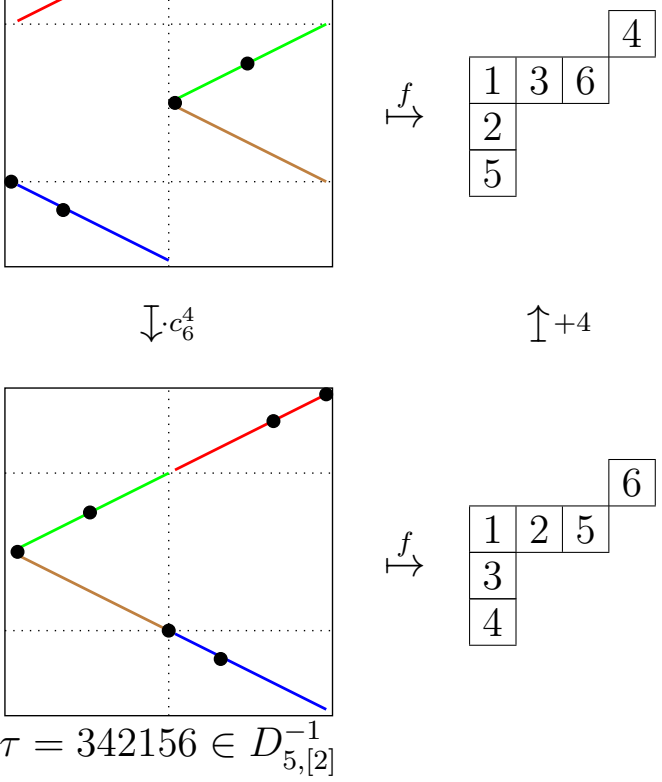

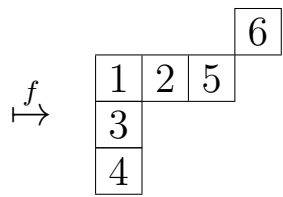

Figure 8: An example of the cDes-preserving bijections $C_{6} D_{5,[2]}^{-1} \stackrel{\phi^{-1}}{\rightarrow} D_{5,[2]}^{-1} C_{6} \stackrel{f}{\rightarrow}$ $\operatorname{SYT}\left(\left(3,1^{2}\right) \oplus(1)\right)$.

\section{References}

[1] R. M. Adin, S. Elizalde and Y. Roichman, Cyclic descents for near-hook and two-row shapes, European J. Combin. 79 (2019), 152-178.

[2] R. M. Adin, I. M. Gessel, V. Reiner and Y. Roichman, Cyclic quasisymmetric functions, arXiv:1811.05440.

[3] R. M. Adin, V. Reiner and Y. Roichman, On cyclic descents for tableaux, Int. Math. Res. Not. IMRN (2018) rny280, https://doi.org/10.1093/imrn/rny280.

[4] R. M Adin and Y. Roichman, Matrices, characters and descents, Linear Algebra Appl. 469 (2015), 381-418.

[5] M. H. Albert, M. D. Atkinson, M. Bouvel, N. Ruskuc and V. Vatter, Geometric grid classes of permutations. Trans. AMS 365 (2013), 5859-5881.

[6] P. Cellini, Cyclic Eulerian elements, European J. Combin. 19 (1998), 545-552.

[7] K. Dilks, T. K. Petersen and J. R. Stembridge, Affine descents and the Steinberg torus, Adv. in Appl. Math. 42 (2009), 423-444.

[8] S. Elizalde and Y. Roichman, Arc permutations. J. Algebraic Combin. 39 (2014), 301-334. 
[9] S. Elizalde and Y. Roichman, Schur-positive sets of permutations via products of grid classes, J. Algebraic Combin. 45 (2017), 363-405.

[10] S. Elizalde and Y. Roichman, On rotated Schur-positive sets, J. Combin. Theory Ser. A 152 (2017), 121-137.

[11] I. M. Gessel, Multipartite P-partitions and inner products of Schur functions, Contemp. Math. 34 (1984), 289-302.

[12] I. M. Gessel and C. Reutenauer, Counting permutations with given cycle structure and descent set, J. Combin. Theory Ser. A 64 (1993), 189-215.

[13] B. Huang, Cyclic descents for general skew tableaux, J. Combin. Theory Ser. A 169 (2020), 45 pages.

[14] O. Pechenik, Cyclic sieving of increasing tableaux and small Schröder paths, J. Combin. Theory Ser. A 125 (2014), 357-378.

[15] B. Rhoades, Cyclic sieving, promotion, and representation theory, J. Combin. Theory Ser. A 117 (2010), 38-76.

[16] R. P. Stanley, Enumerative combinatorics, Vol. 2, Cambridge Studies in Advanced Mathematics, 62, Cambridge Univ. Press, Cambridge, 1999. 